\title{
AVALIAÇÃO DA EFICÁCIA DE RADIOFREQUÊNCIA, PEELING QUÍMICO E MASSOTERAPIA EM ALTERAÇÕES CICATRICIAIS DE PORTADOR DE TROMBOSE VENOSA PROFUNDA: ESTUDO DE CASO
}

\author{
EVALUATION OF THE EFFECTIVENESS OF RADIOFREQUENCY, CHEMICAL \\ PEELING AND MASSOTHERAPY IN SCAR CHANGES OF CARRIER OF DEEP \\ VENOUS THROMBOSIS: CASE STUDY
}

\author{
Paula Bianchetti ${ }^{1}$, Júlia Eduarda Dullius ${ }^{2}$, Rita de Cássia dos Santos ${ }^{2}$, Tatiele Jantsch Bauer ${ }^{2}$, \\ Giovana Sinigaglia ${ }^{2}$ \\ Universidade de Santa Cruz do Sul (UNISC) ${ }^{1}$, Universidade do Vale do Taquari - Univates ${ }^{2}$
}

\begin{abstract}
The Deep Vein Thrombosis (DVT) is a change in blood flow characterized by the formation of clots in the veins that make it difficult and / or block the passage of blood. Due to this alteration, many electrotherapy resources, because they promote some type of peripheral alteration, are contraindicated. The objective of the present study was to evaluate the efficacy of electrotherapeutic resources when associated with chemical peels and massage therapy in a person with DVT in the treatment of postoperative cicatricial alterations, in order to minimize the visible appearance of scars and to improve local sensitivity and malleability. Therefore, nine sessions were conducted with the use of radiofrequency, Light Emitting Diode (LED) $660 \mathrm{~nm}$, chemical peeling, beyond of the tissue release maneuvers for massage therapy, and the complete evaluation was performed at the beginning and at the end of the treatment, from August to November, 2018. After nine sessions, the results this study have shown to be satisfactory, with improvement in the hyperpigmentation of the scars, decrease of the fibrotic aspect and improvement of the aspect of the scar. It was observed that resource used is effective and do not cause evident risks in the case of the person with DVT.
\end{abstract}

Key words: Venous Thrombosis, Scar, Radiofrequency, LED, Chemical Peeling.
Resumo

A Trombose Venosa Profunda (TVP) é uma alteração do fluxo sanguíneo caracterizada pela formação de coágulos nas veias que dificultam e/ou bloqueiam a passagem do sangue. Devido esta alteração, muito recursos de eletroterapia, por promoverem algum tipo de alteração periférica, são contraindicados. O objetivo do presente trabalho foi avaliar a eficácia de recursos eletroterápicos quando associados à peelings químicos e massoterapia, em indivíduo portador de TVP no tratamento de alterações cicatriciais pós operatórias, com a finalidade de minimizar o aspecto visível das cicatrizes $e$ melhorar a sensibilidade e maleabilidade local. Para tanto, foram realizadas nove sessões com o uso de radiofrequência, Light Emitting Diode (LED) $660 \mathrm{~nm}$, peeling químico, além das manobras de liberação tecidual de massoterapia, sendo a avaliação completa realizada no início e no fim do tratamento, no período de agosto a novembro de 2018. Após nove sessões, os resultados deste estudo mostraram-se satisfatórios, com melhora na hiperpigmentação das cicatrizes, diminuição do aspecto fibrótico e melhora do aspecto da cicatriz. Observou-se que os recursos utilizados são eficazes e não causam riscos evidentes no caso do indivíduo portador de TVP.

Palavras chave: Trombose Venosa, Cicatriz, Radiofrequência, LED, Peeling Químico. 


\section{Introdução}

A Trombose Venosa Profunda (TVP) é caracterizada pela formação de coágulos sanguíneos (trombos) nas veias que podem dificultar ou bloquear a passagem de sanguei, impossibilitando a utilização de muitos recursos de eletroterapia disponíveis, que em sua maioria promovem algum tipo de alteração da circulação periférica, seja por induzir uma inflamação, vibração ou calor, efeitos esses que são almejados para o tratamento de alterações cicatriciais ${ }^{2}$.

O mecanismo de cicatrização de feridas após uma lesão é um processo complexo que ocorre em organismos multicelulares. Nos mamíferos, a resposta típica a uma lesão é a formação de cicatriz fibrótica, que restabelece a integridade e a função do tecido ${ }^{3}$.

Para otimizar o processo de cicatrização, favorecendo assim um aspecto estético mais harmônico, diferentes recursos estéticos podem ser utilizados, logo os recursos de eletroterapia utilizados para estas alterações atuam na mobilidade tecidual, através do aumento de fibroblastos e reorganização das fibras elásticas. O peeling químico atua como queratolítico, despigmentante e renovador celular e a liberação tecidual com manobras de massoterapia atua como organizadora dos feixes de colágeno através do realinhamento e tração ${ }^{4}$.

O mecanismo de ação da radiofrequência (RF) é baseado em uma corrente elétrica oscilante forçando colisões entre moléculas carregadas e íons, que são transformados em calor. $\mathrm{O}$ aquecimento tecidual gerado por RF tem diferentes efeitos biológicos e clínicos, dependendo da profundidade do tecido alvo, da frequência utilizada e do resfriamento específico da derme e da epiderme. A profundidade de penetração da energia de RF é inversamente proporcional à frequência. Consequentemente, frequências mais baixas de RF são capazes de penetrar mais profundamente ${ }^{5}$.

A terapia luminosa de baixa intensidade (o LED) emite fótons que são absorvidos pelas células eucariotas, mais especificamente, pelo citocromo e oxidase. Com isso, ocorre um aumento na síntese de colágeno e proliferação dos fibroblastos pela estimulação da oxigenação e nutrição tecidual ${ }^{6}$. A utilização do LED 660nm (luz vermelha), por sua ótima interação com as células, atua como reparador tecidual, contribuindo também para uma boa cicatrização e analgesia. Isso se dá através do aumento de aporte sanguíneo, que tem como consequência a proliferação celular, intensificando a síntese de colágeno e elastina?.

O acúmulo de componentes da matriz extracelular (MEC), principalmente colágeno, e aumento da rigidez tecidual são características comuns da fibrose ${ }^{8}$. A prevenção e o tratamento da fibrose e a formação de aderências por meio de alongamento e mobilização manual são opções simples e econômicas para um dos problemas mais comuns após procedimentos cirúrgicos ${ }^{9}$.

Os peelings químicos são importantes aliados no tratamento de afecções cutâneas, dentre os diferentes tipos de compostos estão os alfa hidroxiácidos (AHAs) que são ácidos carboxílicos, em concentrações baixas reduzem a adesão dos corneócitos, promovendo efeito esfoliativo ${ }^{10}$.

O ácido glicólico é extraído da cana de açúcar, apresenta baixo peso molecular e por esse motivo têm facilidade em penetrar as camadas da pele, sendo o esfoliante mais potente entre os AHAs, com importante ação regeneradora ${ }^{11}$.

O ácido mandélico é um composto derivado da hidrólise de amêndoas amargas, possui menos permeação quando comparado ao ácido glicólico, pois apresenta maior peso molecular. É um peeling muito versátil, tem ação antiinflamatória, anti séptica, hidratante, regenerador da epiderme, queratolítico, é utilizado para prevenir o envelhecimento cutâneo ${ }^{12}$.

$\mathrm{O}$ ácido ascórbico (Vitamina C) é importante na cicatrização das feridas, essencial na síntese de colágeno (atua como co-fator para as enzimas lisil e prolil hidroxilases) e tem sido utilizado também como clareador cutâneo, inibindo a tirosinase ${ }^{13}$.

O objetivo do presente trabalho foi avaliar a eficácia de recursos eletroterápicos quando associados à peelings químicos e massoterapia, em indivíduo portador de TVP no tratamento de alterações cicatriciais pós operatórias, com a finalidade de minimizar o aspecto visível das cicatrizes e melhorar a sensibilidade e maleabilidade local.

\section{Método}

Trata-se de um estudo de caso qualitativo, quantitativo, exploratório, do tipo estudo de caso, por amostra intencional, que foi realizado no Laboratório de Estética Corporal da Universidade do Vale do Taquari - UNIVATES (Lajeado-RS, Brasil) no período de agosto à novembro de 2018, tendo o participante assinado o Termo de 
Consentimento Livre e Esclarecido - TCLE e o Termo de Uso de Imagem.

\section{Descrição do Indivíduo}

Foi selecionado um indivíduo, do sexo masculino, com idade de 36 anos, professor universitário, com $71,8 \mathrm{Kg}$ e $1,66 \mathrm{~m}$ (IMC = $26,06 \mathrm{Kg} / \mathrm{m} 2$ ). Fazia uso de anticoagulante (Xarelto) e antidepressivos (Cloridrato de Sertralina e Carbonato de Lítio). Apresentava reação alérgica a Dipirona. Sem alteração de pressão arterial, problemas cardíacos, diabetes, alterações de tireoide, epilepsia, nem portador de metais no corpo e sem antecedentes oncológicos.

Realizou em 2007 procedimento cirúrgico de septoplastia com cauterização de cornetos internos. Devido a trauma por arma de fogo na região de flanco direito com exteriorização incompleta em coxa esquerda e laceração de órgãos internos, em dezembro de 2017, foi submetido a laparotomia exploratória para rafia de intestino e bexiga, além de inguinotomia esquerda onde houve o rompimento total da veia femoral.

Para reconstrução do fluxo venoso, foi necessária a ligação da veia femoral com a safena interna. Não foi possível a ligação na safena externa (primeira opção), devido a mesma também estar lacerada, precisando ser interrompida por ligadura pois já havia comprometimento do fluxo venoso. Sendo este o principal motivo para o desenvolvimento da Trombose Venosa Profunda.

No período do tratamento proposto para este estudo, o indivíduo praticava atividades físicas três vezes por semana (academia), ingeria diariamente dois litros de água, não tinha alimentação balanceada com frequente ingestão de frituras, mas pouca ingestão de doces. Seu trânsito intestinal era regular, não era fumante e ingeria álcool socialmente.

\section{Técnicas terapêuticas utilizadas}

Para a realização do estudo, foram utilizados os seguintes equipamentos:

- Radiofrequência (KLD, Hertix), manopla bipolar com uso de gel para deslizamento. Os parâmetros de utilização do equipamento foram: $2400 \mathrm{KHz}$, dose de $36 \%$, temperatura de 35 o C $( \pm 1 C)$ até aquecer levemente o tecido.

- LED (Fluence - HTM), 660nm, com energia de 3 Joules, 30 segundos por disparo, com aplicação de forma pontual.
- Peeling químico de ácido glicólico em combinação com o ácido mandélico. Aplicou-se da seguinte forma: Higienização com água micelar (L'Oreal); seguido de delipidação com álcool 70\%; aplicação de ácido glicólico (Bio Age) por 20 minutos; aplicação de ácido mandélico (Samana) por 15 minutos; aplicação de Máscara Facial Clareadora Fogo (Samana) por 20 minutos; aplicação do Gel Creme Vitamina C (Bio Age).

- Manobras de liberação tecidual de massoterapia com movimentos de amassamento, rolamento, fricção e deslizamento realizado sobre a cicatriz em movimentos de vai e vem com os dedos indicadores (Figura 1). Foram aplicados em torno de 10 movimentos em cada local, em todo o comprimento da cicatriz.

Todas as técnicas foram aplicadas sob as três cicatrizes.

\section{Avaliação}

A avaliação das cicatrizes foi realizada através da ficha de avaliação adaptada da Universidade do Vale do Taquari - UNIVATES, que consiste de identificação, anamnese e exame físico - representado pela observação, além do teste Estesiométrico. Além disso, foram realizados registros fotográficos em decúbito dorsal e em posição ortostática.

A primeira avaliação do indivíduo foi realizada no dia 06 de agosto de 2018, tendo sido utilizados os seguintes recursos:

- Kit teste de sensibilidade (Estesiômetro) onde foi observado que na parte inferior da cicatriz abdominal e na parte superior da cicatriz inguinal, o participante sente o monofilamento laranja (10 gf) e nas demais regiões, sente o monofilamento azul (0,2 gf);

- Peso e altura: foi utilizada balança (Welmy) para pesagem e verificação da altura do indivíduo;

- Fotografia para o registro: foi utilizada câmera fotográfica (Sony DSC-HX300), com o auxílio de um tripé.

Os critérios clínicos analisados foram: cicatriz hiperpigmentada e fibrose nas regiões abdominal, inguinal (esquerda) e femoral (esquerda). As cicatrizes eram decorrentes de pós operatório de Laparotomia Exploratória (abdômen), Inguinotomia (inguinal) e retirada de projétil (femoral).

\section{Procedimento experimental}

A Tabela 1 apresenta os procedimentos que foram realizados em cada atendimento. 
Figura 1: Ilustração das manobras de liberação tecidual de massoterapia. A linha preta é uma representação da cicatriz.

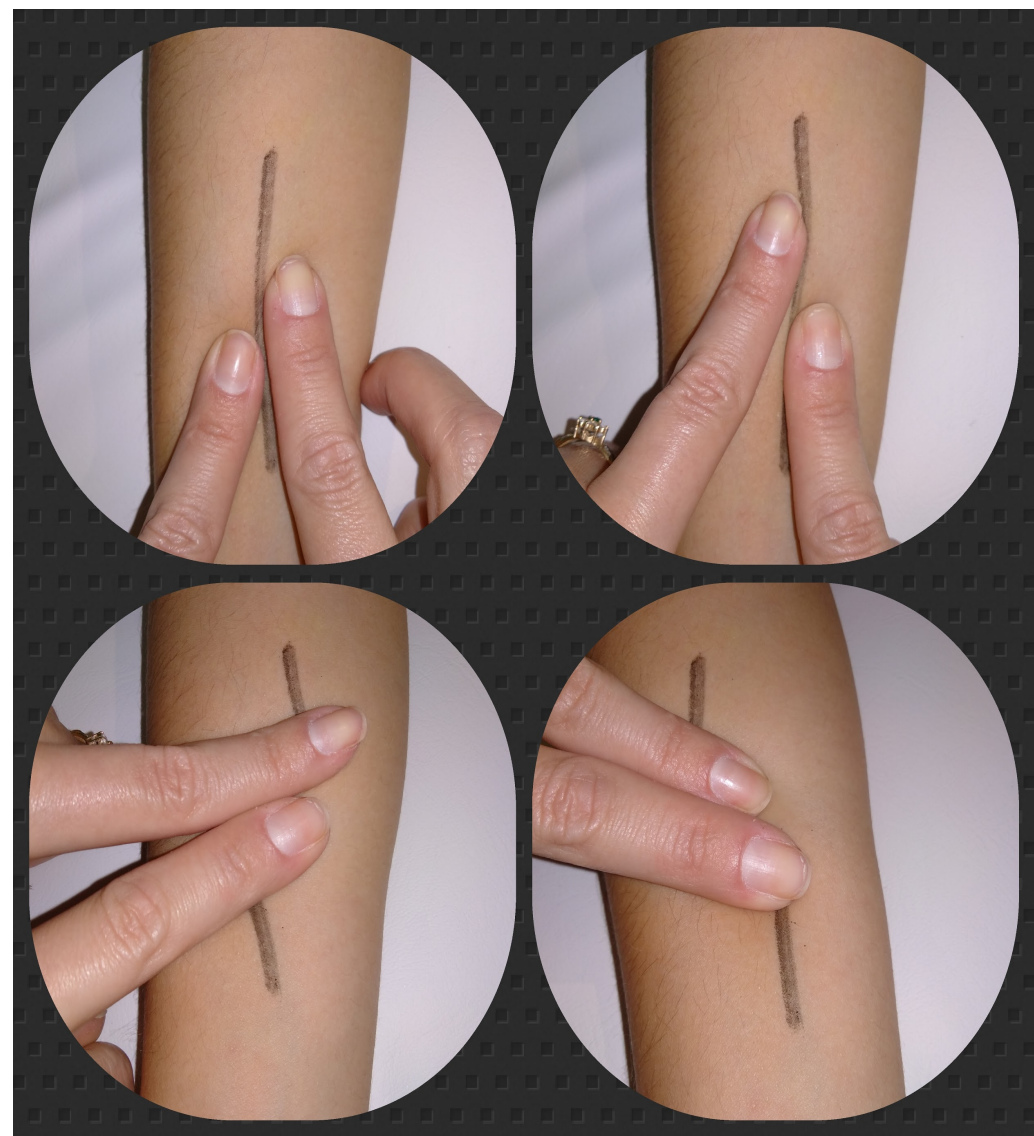

Tabela 1: Procedimentos realizados por atendimento

\begin{tabular}{|c|c|l|}
\hline ATENDIMENTO & DATA & \multicolumn{1}{c|}{ PROCEDIMENTO } \\
\hline $\mathbf{1}$ & $29 / 08 / 2018$ & Radiofrequência; Manobras de Massoterapia; LED 660nm; Vitamina C \\
\hline $\mathbf{2}$ & $12 / 09 / 2018$ & Radiofrequência; Manobras de Massoterapia; Vitamina C \\
\hline $\mathbf{3}$ & $19 / 09 / 2018$ & Radiofrequência; Manobras de Massoterapia; LED 660nm; Vitamina C \\
\hline $\mathbf{4}$ & $01 / 10 / 2018$ & Radiofrequência; Manobras de Massoterapia; Vitamina C \\
\hline $\mathbf{5}$ & $03 / 10 / 2018$ & Ácido Glicólico; Ácido Mandélico Máscara de Ácido Kójico; Vitamina C \\
\hline $\mathbf{6}$ & $08 / 10 / 2018$ & Radiofrequência; Manobras de Massoterapia; LED 660nm; Vitamina C \\
\hline $\mathbf{7}$ & $22 / 10 / 2018$ & Ácido Glicólico; Ácido Mandélico; Máscara de Ácido Kójico; Vitamina C \\
\hline $\mathbf{8}$ & $29 / 10 / 2018$ & Radiofrequência; Manobras de Massoterapia; LED vermelho; Vitamina C \\
\hline $\mathbf{9}$ & $05 / 11 / 2018$ & Ácido Glicólico; Ácido Mandélico; Máscara de Ácido Kójico; Vitamina C \\
\hline
\end{tabular}

Os atendimentos foram totalizados em nove sessões. Logo após o término do tratamento, foi realizado um registro fotográfico final, obedecendo aos mesmos padrões da avaliação fotográfica inicial.

Para o home care, foi solicitado ao paciente que utilizasse diariamente o creme Dermosoft Revitalize (Dermo Vit C, Extratos da Terra) 
composto por vitamina $\mathrm{C}$ e resveratrol nanoencapsulados, extrato de ferula foetida e extrato de kakadu, com o objetivo de promover hidratação da pele, clareamento das hiperpigmentações e melhora da elasticidade através da permeação profunda provocada.

\section{Questões éticas}

Para que o paciente pudesse participar do estudo, foram levados em consideração os critérios de inclusão: sexo masculino, idade entre 30 e 40 anos, apresentar fibrose, hiperpigmentação cicatricial e assinar o Termo de Consentimento Livre e Esclarecido - TCLE e o Termo de Uso de Imagem.

Os critérios de exclusão foram: doenças cardiovasculares graves; próteses no quadril ou nos joelhos; hérnia de disco, discopatia ou espondilolistese, inflamações agudas; marcapasso, úlceras, pessoas com deslocamento da retina; labirintite; endopróteses ou implantes metálicos no local de aplicação, neoplasias; processos infecciosos, processos inflamatórios agudos; tecido isquêmico e lesões tuberculosas ativas.

\section{Análise dos dados}

Os dados foram descritos através da análise do questionário de satisfação - adaptadoii e registro fotográfico comparativo, assim como as variáveis categóricas foram expressas através de medidas e percentuais.

\section{Resultados e Discussão}

O participante iniciou 0 tratamento pesando $71,8 \mathrm{Kg}$ e medindo $1,66 \mathrm{~m}$ e terminou com $72,3 \mathrm{Kg}$ e $1,66 \mathrm{~m}$ (IMC $=26,2 \mathrm{Kg} / \mathrm{m} 2$ ). Estes resultados indicam que o tratamento não alterou significativamente, peso e altura do mesmo, demonstrando não interferir nos hábitos de vida diária do indivíduo, tendo em vista o objetivo do estudo que foi avaliar o aspecto cicatricial, após intervenção estética em pós-operatório tardio. A Figura 2 apresenta o registro fotográfico da cicatriz abdominal $(1 A)$, inguinal $(2 A)$ e femoral (3A) antes e depois (1B, 2B, 3B, respectivamente) do tratamento proposto nesta pesquisa.

É possível observar, a partir dos registros fotográficos, que houve melhora significativa na hiperpigmentação das três cicatrizes. É provável que a associação de despigmentantes utilizada no peeling químico foi eficaz, além da contribuição do efeito clareador da vitamina C presente no home care e do LED vermelho. Em um estudo realizado ${ }^{15}$ avaliou a associação do ácido ascórbico (AA) 5\% vs. hidroquinona $4 \%$ em 16 pacientes com melasma, concluiu que o lado que teve $O A A$ aplicado teve resultados excelentes em dois pacientes, bons em oito, moderada em quatro, e leve em dois.

Gonchoroski e Côrrea ${ }^{16}$ realizaram estudo comparando a ação despigmentante de três formulações no tratamento de hipercromia pós inflamatória contendo hidroquinona (4\%), ácido retinóico $(0,05 \%)$, ácido kójico $(8 \%)$ e ácido glicólico $(6 \%)$ concluiu que todas as formulações tiveram ação despigmentante, porém a hidroquinona associada ao ácido retinóico teve ação mais eficaz a curto prazo, sugerindo a associação de ácido kòjico e ácido glicólico para tratamentos à longo prazo.

O uso da vitamina C apresenta melhora significativa na redução da pigmentação ${ }^{17}$. De acordo com Umbria et al ${ }^{18}$ a vitamina $C$ é indispensável para síntese de colágeno e a deficiência desta vitamina desorganiza a ultraestrutura do fibroblasto. É capaz de estimular a proliferação celular, bem como a síntese de colágeno pelos fibroblastos dérmicos, superando a proliferação reduzida destes fibroblastos na pele envelhecida, mostrando benefício no processo de cicatrização.

Estudo realizado por Lee et al $^{19}$ apresenta redução global da melanina com o uso da luz vermelha $(633+6 \mathrm{~nm})$. Os níveis de melanina diminuíram significativamente $(p<0,005)$, após a irradiação da luz vermelha. O clareamento da pele foi relatado por 14 dos 24 indivíduos após o período de tratamento.

Mpofana e Ramhurry ${ }^{20}$ em uma pesquisa qualitativa e quantitativa, concluíram que o LED $(633 \mathrm{~nm})$ é eficaz para o tratamento de melasma, contribuindo para a uniformização do tom da pele, através da inibição da tirosinase. Além disso, o uso do LED vermelho apresenta-se como uma excelente alternativa para a cicatrização e reparação tecidual já que não causa efeitos colaterais $^{21}$

Em relação à fibrose, caracterizada como queixa principal, através das respostas obtidas pelo Questionário de Satisfação, foi possível identificar melhora no aspecto fibrótico das cicatrizes, das atividades diárias do indivíduo e sua satisfação em relação aos resultados. Além disso, o participante relatou aspectos positivos e atividades que, após os atendimentos, tornaramse possíveis de serem realizadas: movimentações laterais, distensionamento na região abdominal, diminuição do desconforto ao sentar e do atrito da cicatriz com a calça jeans.

Estudo realizado por Altomare e Monte- 
alto- $\operatorname{costa}^{9}$ com o uso de mobilização tecidual manual em camundongos que sofreram uma lesão microcirúrgica, constatou que a estimulação mecânica através da mobilização reduziu a fibrose subcutânea.

Figura 2: Registro fotográfico da cicatriz abdominal (1), inguinal (2) e femoral (3). Antes (A), em 06/08/2018, e depois (B) em 05/11/2018, após a realização de 9 sessões da terapia proposta.
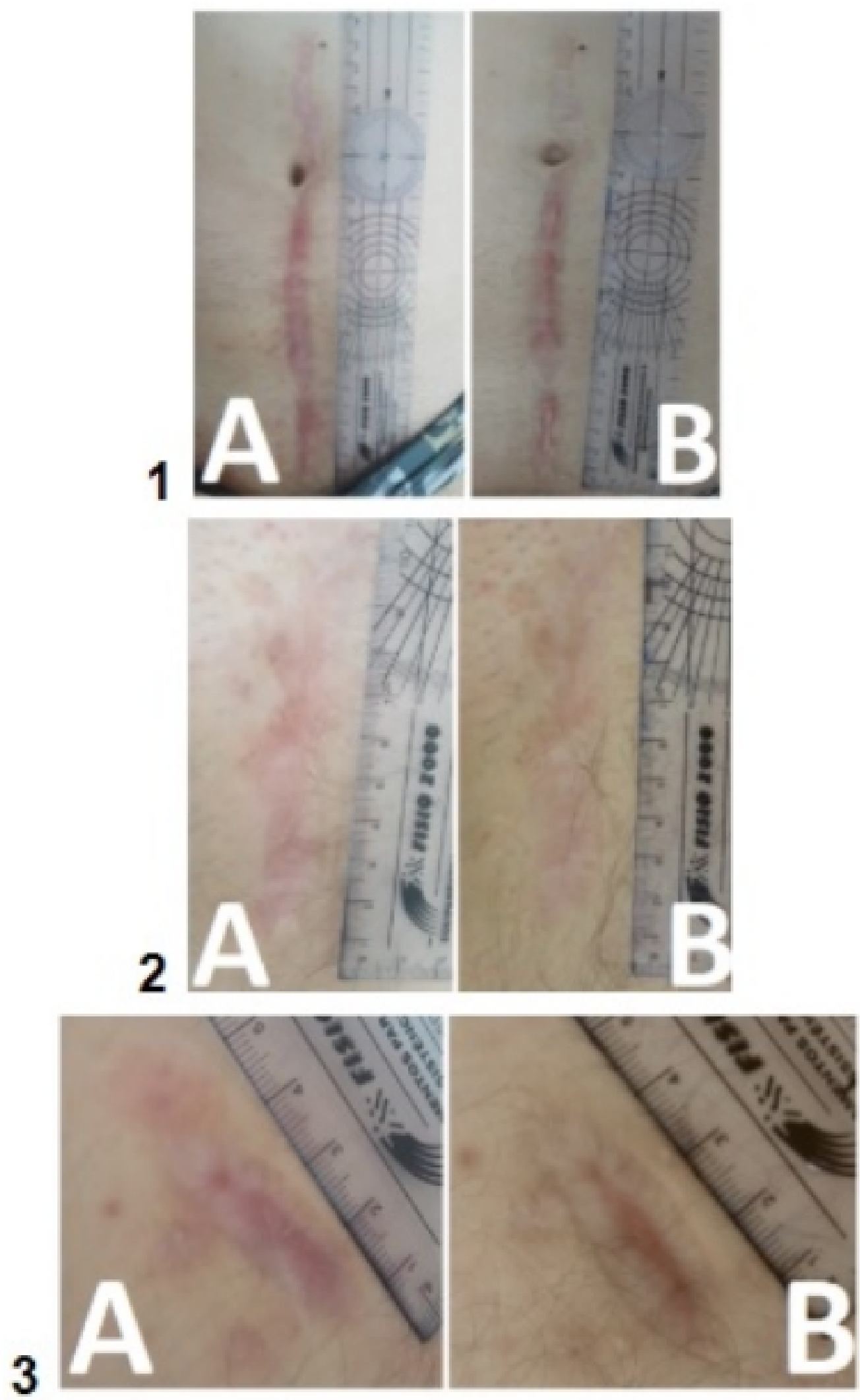
Em relação à sensibilidade cutânea foi possível constatar que houve melhora em relação à sensibilidade das regiões cicatriciais, conforme dados da tabela 2.

Tabela 2: Avaliação da sensibilidade das regiões tratadas, antes e depois da aplicação terapêutica, sendo apresentados os valores em grama-força (gf).

\begin{tabular}{|c|c|c|}
\hline REGIÃO & Antes (gf) & Depois (gf) \\
\hline Abdominal Inferior & 10,0 & 0,2 \\
\hline Abdominal Superior & 0,2 & 0,2 \\
\hline Inguinal Inferior & 0,2 & 0,2 \\
\hline Inguinal Superior & 10,0 & 2,0 \\
\hline Femoral & 0,2 & 0,2 \\
\hline
\end{tabular}

Observa-se importante melhora da sensibilidade tátil na região abdominal inferior e inguinal superior, áreas estas que estavam significantemente afetadas. A perda de sensibilidade pode afetar nas atividades diárias e promover lesão por excesso de atrito nos tecidos afetados $^{22}$.

Em relação à extensão da parte hiperpigmentada das lesões (Tabela 3), foi possível observar que houve diminuição de tamanho de todas as cicatrizes.

Tabela 3: Comparativo da extensão das cicatrizes ante e depois da aplicação terapêutica.

\begin{tabular}{|c|c|c|}
\hline CICATRIZ & Antes $(\mathrm{cm})$ & Depois $(\mathrm{cm})$ \\
\hline Abdominal & 23 & 20,5 \\
\hline Inguinal & 10 & 9 \\
\hline Femoral & 4,5 & 2 \\
\hline
\end{tabular}

Os resultados obtidos demonstram interessante redução da extensão das cicatrizes, com redução das cicatrizes de $10,86 \%$ na região abdominal, $10 \%$ na inguinal e $55,55 \%$ na localização femoral.

\section{Conclusão}

A partir deste estudo de caso, voltado para o tratamento de alterações cicatriciais pós operatórias (hiperpigmentação e fibrose) que ocorreu de agosto à novembro de 2018, utilizando radiofrequência e LED vermelho associado à liberação tecidual com manobras de massoterapia e peeling químico, pôde-se evidenciar, que os resultados obtidos foram satisfatórios.

É importante salientar que os recursos foram aplicados apenas uma vez por semana o que reduz a qualidade dos resultados pelo número limitado de sessões em virtude do tempo despendido para o tratamento, que foi de quatro meses. Logo, torna-se importante a continuidade do tratamento proposto, havendo a necessidade de maior número de sessões a serem realizadas, para obter melhores resultados. Além disso, através do comparativo realizado com outros estudos, observou-se que outros ativos despigmentantes poderiam ter sido utilizados para obter resultados mais eficazes.

Este estudo foi importante para demonstrar que há recursos disponíveis e eficazes para um paciente portador de Trombose Venosa Profunda, uma alteração que é sempre considerada uma contraindicação para a maioria dos tratamentos realizados pelo profissional de Estética.

\section{Referências}

1. Panucci CJ, Obi AT, Timmins $B H$, Cochran AL. Venous Thromboembolism in Patients with Thermal Injury. Clinics in Plastic 
Surgery [revista em internet] 2017 julho. [acesso 16 de agosto de 2018; 44(3). Disponível: $<$ https://www.plasticsurgery.theclinics.com/articl e/S0094-1298(17)30016-0/fulltext>.

2. Salgado ASI. Eletrofisioterapia: manual clínico. São Paulo: Andreoli, 2013.

3. Duscher D, Maan ZN, Wong VW, Rennert RC, Januszyk $M$, Rodrigues $M, H u M$, Whitmore EAJ, Whittam AJ, Longaker MT, Gurtner GC. Mechanotransduction and fibrosis. Journal of Biomechanics [revista em internet] 2014 junho. [acesso 24 de agosto de 2018]; 47(9). Disponível em

<https://www.sciencedirect.com/science/article/ pii/S0021929014002012>.

4. KEDE, M. P. V; SABATOVICH, O. Dermatologia estética. São Paulo: Atheneu, Ed. 3, 2015.

5. Beasley KL, Weiss RA. Radiofrequency in Cosmetic Dermatology. Dermatol Clin [revista em internet] 2014 janeiro. [acesso 16 de agosto de 2018]; 32(1). Disponível em: <https://www.derm.theclinics.com/article/S0733 -8635(13)00096-X/fulltext>.

6. Karu TI. Cellular mechanisms of low power laser therapy: new questions. Laser Application in Medicine, Biology, and Environmental Science, 2003. [acesso em 13 de dezembro de 2018]. Disponível em: $<$ https://www.researchgate.net/publication/242 397681_CELLULAR_MECHANISMS_OF_LOW_PO WER_LASER_THERAPY_NEW_QUESTIŌNS>.

7. Ferreira CLR, Nicolau RA, Oliveira MA, Costa DR, Prianti Junior ACG. Efeito da terapia LED $(\lambda=945 \pm 20 \mathrm{~nm})$ de baixa intensidade sobre tecido epitelial de ratos diabéticos em processo de reparo. Rev. Bras. Eng. Bioméd. [revista em internet] 2013 dezembro. [acesso 21 de agosto de 2018]; 29(4). Disponível em: <http://rbejournal.org/doi/10.4322/rbeb.2013.0 40>.

8. Cutroneo KR. How is Type I procollagen synthesis regulated at the gene level during tissue fibrosis. Journal of Cellular Biochemistry [revista em internet] 2003 agosto. [acesso 27 de agosto de 2018]; 90(1). Disponível em: $<$ https://onlinelibrary.wiley.com/doi/abs/10.100 2/jcb.10599>.

9. Altomare $\mathrm{M}$, Monte-Alto-Costa A. Manual Mobilization of Subcutaneous Fibrosis in Mice. Journal of Manipulative and Physiological Therapeutics [revista em internet] 2018 junho. [acesso 24 de agosto de 2018]; 41(5). Disponível em:

<https://www.sciencedirect.com/science/article/ pii/S0161475416300094?via\%3Dihub>

10. Alam M, Gladstone HB, Tung RC.
Dermatologia cosmética. Rio de Janeiro: Elsevier, 2010.

11. Pereira MFL. Cosmetologia.1 ed. São Caetano do Sul: Difusão editora, 2013.

12. Gomes RK, Damasio MG. Cosmetologia: descomplicando os princípios ativos. 3 ed. São Paulo: Livraria Médica Paulista Editora, 2019.

13. Manela-Azulay $M$, Lacerda CAM, Perez MA, Filgueira AL, Cuzzi T. Vitamina C*. An. bras. Dermatol. [revista em internet] 2003 maio/junho. [acesso 11 de outubro de 2018]; 78(3). Disponível em:

<http://www.scielo.br/pdf/abd/v78n3/16303.pdf $>$

14. Suda EY, Uemura MD, Velasco E. Avaliação da satisfação dos pacientes atendidos em uma clínica-escola de Fisioterapia de Santo André, SP. Fisioterapia e Pesquisa [revista em internet] 2009 abril-junho. [acesso 25 de outubro de 2018]; 16(2). Disponível em: <http://www.scielo.br/pdf/fp/v16n2/06.pdf>.

15. Espinal-Perez LE, Moncada B, Castanedo-Cazares JP. Um estudo duplo-cego randomizado de ácido ascórbico a $5 \%$ vs. hidroquinona a $4 \%$ em melasma. International Journal of Dermatology [revista em internet] 2004 agosto. [acesso 24 de outubro de 2018]; 43(8). Disponível em $<$ https://onlinelibrary.wiley.com/doi/full/10.111 1/j.1365-4632.2004.02134.x>.

16. Gonchoroski DD, Côrrea GM. Tratamento de hipercromia pós-inflamatória com diferentes formulações clareadoras. Infarma [revista em internet] 2005. [acesso 18 de outubro de 2018]; 17(3/4). Disponível em: <http://cff.org.br/sistemas/geral/revista/pdf/17/ tratamento_de_hipercromia.pdf>.

17. Steiner D, Feola C, Bialeski N, Silva FAM. Tratamento do melasma: revisão sistemática. Surgical \& Cosmetic Dermatology [revista em internet] 2009 maio. [acesso 25 de outubro de 2018]; 1(2). Disponível em: <http://www.redalyc.org/html/2655/265520997 $008 />$.

18. Umbria KFT, Schwarz SBF, Pinheiro $H$. Estudo comparativo da aplicação da microcorrente contínua filtrada e do ácido ascórbico no tratamento de estrias. Fisioterapia Ser [revista em internet] 2006 junho. [acesso 24 de outubro de 2018]; 1(2). Disponível em: <https://docplayer.com.br/40766317-Estudocomparativo-da-aplicacao-da-microcorrentecontinua-filtrada-e-do-acido-ascorbico-notratamento-de-estrias.html>.

19. Lee SY, You CE, Park, MY. Blue and red light combination LED phototherapy for acne vulgaris in patients with skin phototype IV. Lasers 
in Surgery and Medicine [revista em internet] 2006 novembro. [acesso 07 de novembro de 2018]; 39(2). Disponível em <https://onlinelibrary.wiley.com/doi/abs/10.100 2/lsm.20412>.

20. Mpofana N, Ramhurry C. An Investigation into the Effectiveness of Light Emitting Diodes on Treating Melasma on Skin Type VI. American Journal of Dermatology and Venereology [revista em internet] 2014. [acesso 07 de novembro de 2018]; 3(3). Disponível em: <http://article.sapub.org/10.5923.j.ajdv.2014030 3.01.html>.

21. Dourado KBV, Junior LCC, De Paulo RJF, Gomes AC. Ledterapia: uma nova perspectiva terapêutica ao tratamento de doenças da pele, cicatrização de feridas e reparação tecidual. Ensaios e Ciência [revista em internet] 2011 agosto. [acesso 20 de agosto de 2018]; 15(6). Disponível em: $<$ http://www.redalyc.org/articulo.oa?id=260242 21017>.

22. Tebecherani AJ. Histologia básica cutânea. In: Malagutti W, Kakihara CT. Curativos, estomias e dermatologias: uma abordagem multiprofissional. 3 Ed. São Paulo: Martinari; 2014.

\section{Endereço para Correspondência}

Universidade de Santa Cruz do Sul (UNISC)

Av. Independência, 2293, Universitário - Santa Cruz do Sul (RS)

CEP.: $96.815-900$

e-mail: pbianchetti@gmail.com

Recebido em 19/05/2019

Aprovado em 19/01/2021

Publicado em 20/01/2021 